This item was submitted to Loughborough's Research Repository by the author.

Items in Figshare are protected by copyright, with all rights reserved, unless otherwise indicated.

\title{
loT driven ambient intelligence architecture for indoor intelligent mobility
}

PLEASE CITE THE PUBLISHED VERSION

https://doi.org/10.1109/DASC/PiCom/DataCom/CyberSciTec.2018.00090

PUBLISHER

(c) IEEE

VERSION

AM (Accepted Manuscript)

LICENCE

CC BY-NC-ND 4.0

\section{REPOSITORY RECORD}

De Silva, Varuna, Jamie Roche, Xiyu Shi, and Ahmet Kondoz. 2019. "lot Driven Ambient Intelligence Architecture for Indoor Intelligent Mobility". figshare. https://hdl.handle.net/2134/35356. 


\title{
IoT Driven Ambient Intelligence Architecture for Indoor Intelligent Mobility
}

\author{
Varuna De Silva, Jamie Roche, Xiyu \\ Shi, and Ahmet Kondoz \\ Institute for Digital Technologies, \\ Loughborough University, London
}

\begin{abstract}
Personal robots are set to assist humans in their daily tasks. Assisted living is one of the major applications of personal assistive robots, where the robots will support health and wellbeing of the humans in need, especially elderly and disabled. Indoor environments are extremely challenging from a robot perception and navigation point of view, because of the ever-changing decorations, internal organizations and clutter. Furthermore, human-robot-interaction in personal assistive robots demands intuitive and human-like intelligence and interactions. Above challenges are aggravated by stringent and often tacit requirements surrounding personal privacy that may be invaded by continuous monitoring through sensors. Towards addressing the above problems, in this paper we present an architecture for "Ambient Intelligence" for indoor intelligent mobility by leveraging IoTs within a framework of Scalable Multi-layered Context Mapping Framework. Our objective is to utilize sensors in home settings in the least invasive manner for the robot to learn about its dynamic surroundings and interact in a human-like manner. The paper takes a semi-survey approach to presenting and illustrating preliminary results from our in-house built fully autonomous electric quadbike.
\end{abstract}

Keywords-ambient, assistive, autonomous, intelligent, mobility, robot, navigation

\section{INTRODUCTION}

Robots that assist humans in their daily tasks will revolutionize the way we live our lives. Personal assistive robots that help the elderly to be more independent [3], or robots that help the blind [4] or robots that assist with rehabilitation [5] are some of the emerging applications of personal assistive robots. The robots will assist humans in many day to day tasks such as personal hygiene, mobility guidance [6], dressing support [7], feeding support [8], and rehabilitation support. The opportunities with assistive robots are numerous and it is expected that personal robots will form an integral part of future smart living environments. However, for assistive robots to become ubiquitous in our daily lives, several challenges need to be tackled.

There are many challenges in assistive robotic technology that need to be overcome. One of the challenges is Robust object recognition. Assistive robots are required to interact with objects of varying size, shape, and degree of mobility and hence must be equipped with the ability to process dynamic sensor data to react adequately and seamlessly adapting to environmental changes. Robust object recognition in real-life settings is still a major challenge that need to be overcome [9]. Human Robot Interaction is another challenge in assistive robots. Knowing what human subjects are doing is a key attribute for robots to plan and execute it's duties. Therefore, human activity recognition forms an integral part of human robot interaction. Human activity recognition involves, posture analysis, gait analysis [6] and skeletal tracking [7]. Another key attribute of human robot interaction is to understand the human emotional behaviour [10]. Knowing the emotional state of a human subject is crucial for a robot to react appropriately (e.g. inform care takers, perform risk mitigation operations). The third challenge is Robot Navigation in Cluttered environments: robot navigation involves safe movement of robot without coming in to collision with obstacles. For example, an autonomous vehicle that drives on its own in road environments has to move safely without having accidents. However, when considering indoor environments navigation become complex due to the clutter in home environments, constantly changing environments due to the position of furniture and due to walls and furniture blocking line-of-sight sensors. Due to these reasons navigation in indoor environments is still an enormous challenge when training personal assistive robots [11]. Finally, one of the most crucial requirements of personal robots, especially within applications of physical assistance, is to deal with unpredictable events [8] and uncertain information [12], i.e. the events for which the robot was not trained before or information that is ambiguous. Robust adaptive control techniques to deal with such situations would be required for safe operation of the robots.

The key to address the above challenges associated with personal assistive robots is to understand the context of the environment in which it operates. Context of a system can be regarded as understanding the situation and making sense of the status of the environment [13]. Low cost connected sensors that continuously monitor various environmental parameters, known as Internet of Things (IoT), enables to capture the context of an environment. To capture the context through IoTs, the data streams generated by the IoTs need to be processed and analysed using machine learning and pattern recognition algorithms. While a personal assistive robot cannot have all the sensors that are necessary to capture useful context, it can rely on IoT in an indoor environment to understand the context and react appropriately. Personal assistive robots that are seamlessly connected to IoTs in home environments give rise to so called Ambient Intelligence. Ambient Intelligence can be defined as the process by which a digital environment (sensor and device network) senses, computes interactions, and assists people in their daily lives [13].

Towards achieving ambient intelligence in indoor environments, this paper presents an architecture for IoT enabled Scalable Multi-layered Mapping for Indoor Robot Control. After presenting the general concept of Scalable Multi-layered Mapping, we will focus on indoor robot navigation as a use-case of this platform. 


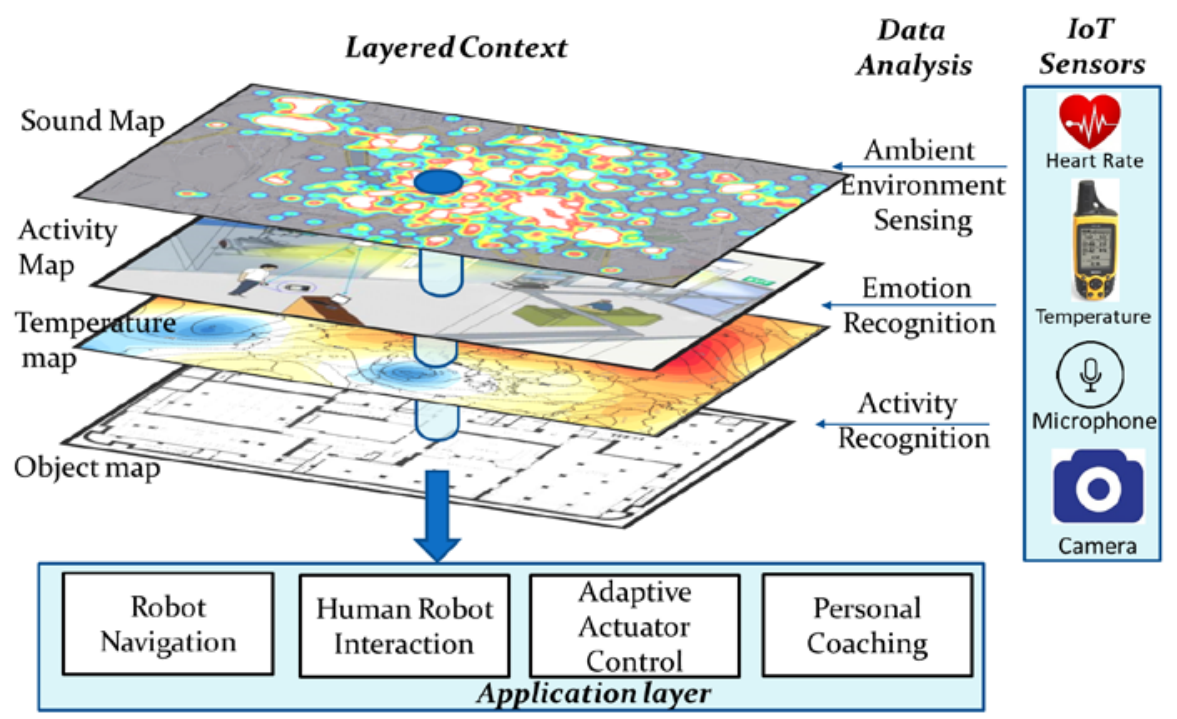

Fig. 1. Architecture for Ambient Intelligence for example use case on intelligent care homes

The rest of this paper is organized as follows: In section II we present the motivation and generalized architecture IoT driven Scalable Multi-layered Mapping for ambient intelligence. Section III presents the architecture for use case on indoor robot navigation followed by a technology review of IoTs for the use case. Section IV describes the architecture for the use case on indoor-robot navigation and introduces our inhouse built autonomous quad-bike and section $\mathrm{V}$ concludes the paper.

\section{IoT ENABLED SCALABLE MULTI-LAYERED MAPPING FOR AMBIENT INTELLIGENCE}

In this section we will describe the proposed Scalable Multilayer Mapping architecture for ambient intelligence. The objective of this architecture is to utilize different kinds of multimodal connected sensors to capture environmental context to enable intelligent control systems. We will explain the components of the architecture by referring to an intuitive use case.

\section{A. Futuristic use case of a care home}

Consider a use case of future care home. In this care home there are different kinds of residents mostly elderly occupants, with different health implications. Multitude of sensors some body worn, such as heart rate and temperature monitors, and some embedded on infrastructure such as CCTV cameras, microphones, temperature monitors and moisture sensors capture data about its environment. There are multiple care-taker robots in this environment that assist human care-takers in various duties: such as personal hygiene, dressing up, feeding, and assisting with mobility. The care home learns personal requirements and behaviour traits of the individuals in the home and keeps a close eye on the emotions of the residents. There are multiple health monitoring applications that utilize body worn sensors of the residents to predict anomalies in health conditions.

\section{B. Requirements Generation}

To realize the above use case several technology components would be necessary. One sensor modality cannot capture all the context. Therefore, multiple sensors that are connected to a central processing system is needed. Secondly, the data captured by different sensors would be of little use, if they are not analysed to capture various parameters of context. Therefore, advanced machine learning algorithms would be necessary to make sense of the sensor data streams. There is a constant need for communication between the sensors, central processing system and the actuators (robots). Therefore, data transmissions have to be light weight, but if necessary the actuators and applications should be able to request for more data. Robots would have specialized sensors, and when it is capturing additional data during an anomalous situation, the captured data needs to be utilized to update the context information. Considering the context layers, the actuators and robots will react appropriately to assist the human subjects.

\section{Architecture Description}

We propose a scalable multi-layer context mapping for ambient intelligence. The architecture for the above use case can be illustrated Fig. 1. The architecture has four layers: Sensing layer, Data Analysis layer, Multi-layered context representation, and Application layer. The data analysis layer constitutes of data pre-processing, data fusion and pattern recognition. The multi-layered context representation will store the captured context to enable different applications. The application layer will communicate with the context representation layer to acquire location dependent context information. To cater to the requirement of scalability for context information, the context representation layer will classifies the context information into different categories according to its complexity, location, resolution, and steadiness.. A requirement for scalability is that different applications would require different levels of context information. For example a dressing robot would not necessarily need context information regarding ambient noise. Furthermore, scalability can be realized as temporal and spatial resolution or based on uncertainty of observations. The data analysis and context storing will happen in centralized data processing system. 


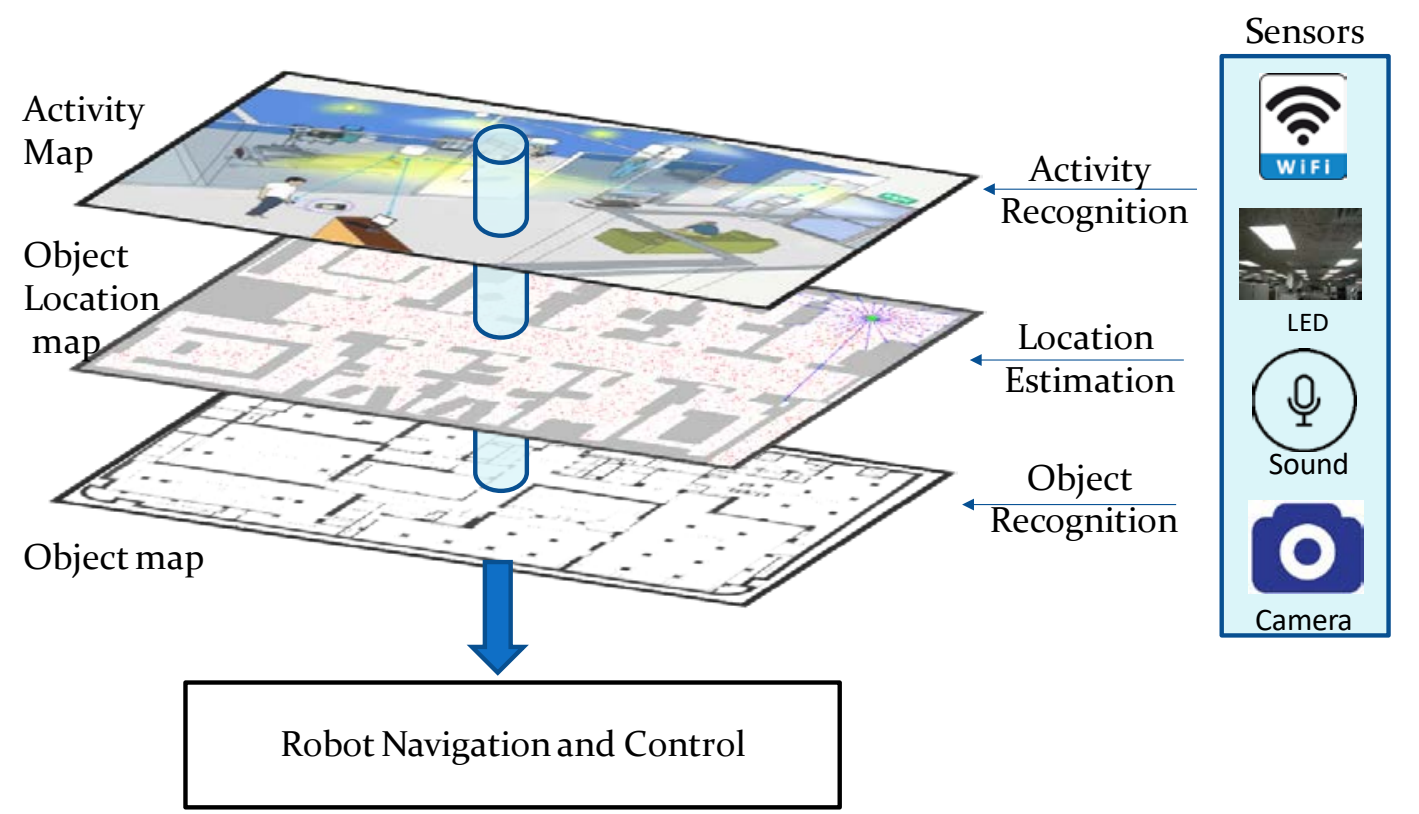

Fig. 2.: Proposed Ambient Intelligence Architecture for Robot Navigation and Localization

In the next section we will illustrate how a scalable multilayered context capturing can be utilized for indoor robot localization and navigation.

\section{AMBIENT INTELLIGENCE DRIVEN INDOOR ROBOT NAVIGATION AND LOCALIZATION}

As discussed in our introduction, there are several challenges to address to make personal assistive robots a commercial success. Among these challenges is the requirement for robust navigation of robots in indoor environments. In this section we will describe how the proposed IoT driven scalable context gathering framework presented in the previous section can be utilized to address the challenge of robot navigation. The section is organized in two parts: the proposed scalable architecture for context gathering derived by the specific challenges in robot navigation and localization, followed by a technology review of IoT for robust context gathering.

\section{A. Challenges in Indoor Robot Navigation and Localization}

Robot navigation involves movement of the robot while avoiding obstacles. The obstacles in a home environment keeps changing its form, appearance, and position. The robot uses its onboard sensors such as cameras and LiDARs to perceive its environment to recognize these obstacles. Furthermore, the robot has to understand the human subjects in its environment and activities they are performing. However, the biggest challenge for robot navigation in indoor environments such as homes is that the line of sight of its sensors are often blocked by walls and furniture. However, for robust robot navigation in home environments, the robot has to effectively see through the walls so that it can detect events such as the elderly falling over in the bathroom.

Another key aspect of robot control is localization of the robot. Localization is the process by which a robot understands its location. While in outdoor environments it is possible to utilize GPS data, in indoor environments GPS is not reliable enough.
Both these problems can be effectively overcome by our proposed scheme on Ambient Intelligence. However, we need to identify appropriate sensors and context to develop an architecture for ambient intelligence to suit this use case. Therefore, in the next subsection, we will perform a technology review to identify suitable sensors and context gathering algorithms..

\section{B. Technology review of suitable IoT sensors for robot navigation and control}

This section is mainly organized in two segments: review of localization algorithms and review of activity recognition.

\section{1) Indoor localization}

GPS based localization schemes are not suitable in indoor environments, due to the significant attenuation of satellite signal power when passing through building material.

Radio Frequency based fingerprinting involves storing the RF characteristics at different locations in an indoor environment in a database and comparing those characteristics (known as fingerprints) of the unknown target location with those stored to find an approximate location of the target. A WiFi based fingerprinting algorithm combining deterministic and probabilistic location estimation for localization of moving IoT targets was proposed in [14]. Radio signal based approach for localization is both a low complexity and a cost effective solution [14]. Fingerprint based positioning technology requires multiple wireless access points (APs) to improve its localization accuracy. To overcome the requirement for multiple wireless APs, authors in [15] proposed an indoor localization system that uses a single Wi-Fi AP to locate terminals by utilizing channel state information (CSI) to compute the direct path length between a single AP and terminals.

Another potential approach for indoor localization could be realized by utilizing the lighting infrastructure of a building. For example, a visible light based location fingerprinting scheme was proposed in [16], where a square wave modulation scheme is employed to allow each of the LED luminaires to be identified by a photodiode-based 


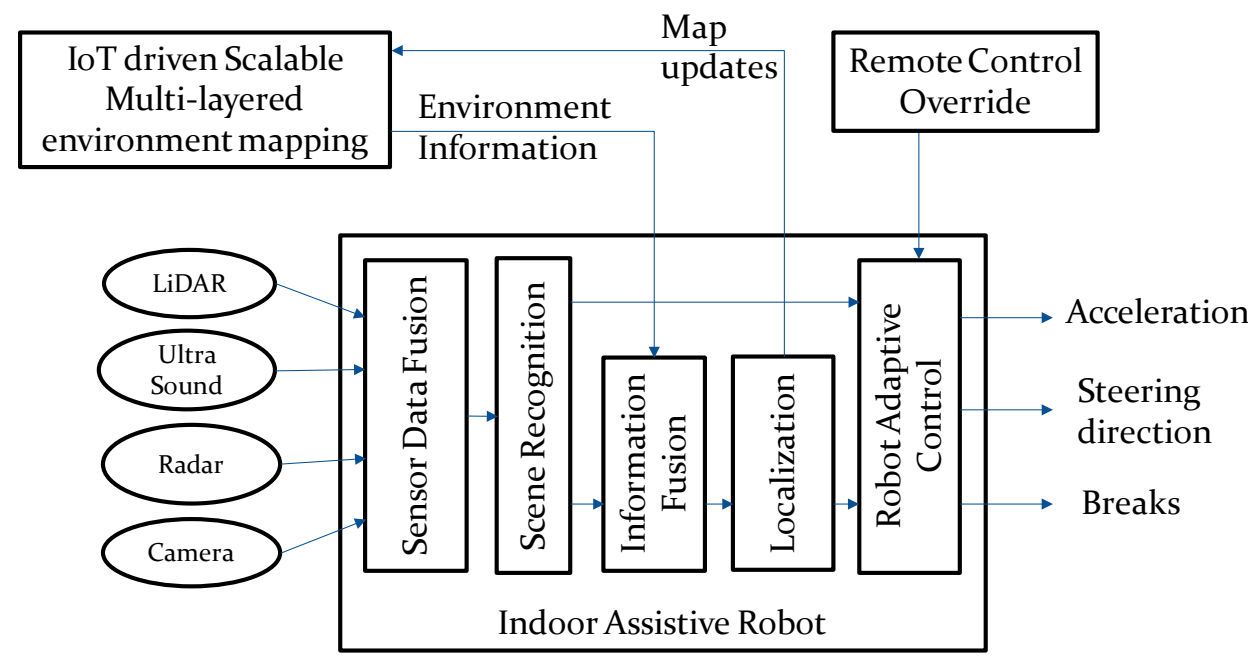

Fig. 3: Integration of Robot with the Ambient Intelligence Architecture

receiver using Fast Fourier Transform (FFT). Several approaches for indoor localization based on visible light communication (VLC) have been proposed in the recent past [17], [18]. While the obvious advantage of VLC for localization includes the use of an existing infrastructure without additional investment, so far such schemes have been demonstrated only in Line of Sight environments.

Both WiFi based localization and VLC based localization requires specific infrastructure to be installed. However, authors in [19] proposed a localization approach that does not need any additional infrastructure other than a smartphone with a microphone. The main hypothesis in [19], was that every location has a distinct set of acoustic characteristics which is a result of constant background noises and modulation of those noises by physical characteristics of the surrounding material, and these characteristics can be utilised effectively for localization based on an acoustic fingerprint. The background noises could be generated by humming of computer fans and air conditioning systems. In [19], acoustic fingerprints are extracted from the training data by filtering out transient sounds from the background noise spectrum, and an SVM classifier is trained on the features. During the online location estimation phase, the acoustic signal characteristics are fed to the classifier for a location estimate.

Another method of indoor localization based on ambient Television (TV) signals was presented in [20], which leverages frequency-selective multipath fading of radio signals that is a physical phenomenon sensitive to receiver's location. However, the lack of TV enabled mobile devices is a major constraint for the widespread use of this technology.

The above indoor localization schemes based on single type of fingerprints is susceptible to the changing environment and on its own tends to have low accuracy in certain settings. Therefore, fusing different fingerprints will enable robust method of localization [21]. Similarly, in [22] a coarse localization result from WiFi fingerprinting was refined using vision based localization by visual feature matching.

\section{2) Activity Recognition}

The use of ambient wireless signals to recognize human activities has received quite a lot of research interest in the recent past. Utilizing wireless signals for activity recognition is particularly interesting due to two main reasons: firstly the wireless signals penetrate through walls and hence can be useful for sensing non-line of sight events (detect falls in bathrooms) and secondly, its researchers may not necessarily need cooperation of subjects due to the lack of body instrumentation (in office environments). Two pioneering efforts were demonstrated using USRP N200 software defined radio units to recognize body gestures [23] and location of humans [24]. To overcome the need for dedicated hardware and firmware updates, [25] proposed an activity recognition system by learning the received signal strength indicator (RSSI) fingerprint of six different activities. In [26], a survey of recent advancements in human activity recognition using WiFi channel status information (CSI) has been conducted. The authors in [26] concluded that techniques such as Long Short Term Memory (LSTM) networks can be effectively utilized to recognize human activities by time series analysis of CSI. However, the robust detection of activities in different dynamic environments, and how to identify the behaviours of multiple users remain a significant challenge.

Various different sensors such as smart phone sensors, cameras, depth sensors and LiDARs have all been investigated for human activity recognition. An extensive survey of IoT based human activity recognition can be found here.

\section{The proposed Ambient Intelligence Architecture for Robot Navigation}

Based on the review of related IoT sensors, we can derive an architecture for robot navigation and control as illustrated in Fig. 2. In this architecture, different sensors such as cameras, noise (sound), light and WiFi sensing can be utilized. The sensor data streams can be analysed to identify objects and humans, recognize human activities and to calculate the location of individual objects relative to the robot. These contexts are gathered in scalable layers, and can be utilized to control the navigation of the robot in indoor environment. In the next section, we will illustrate our inhouse built robotic platform that can benefit from this architecture. 


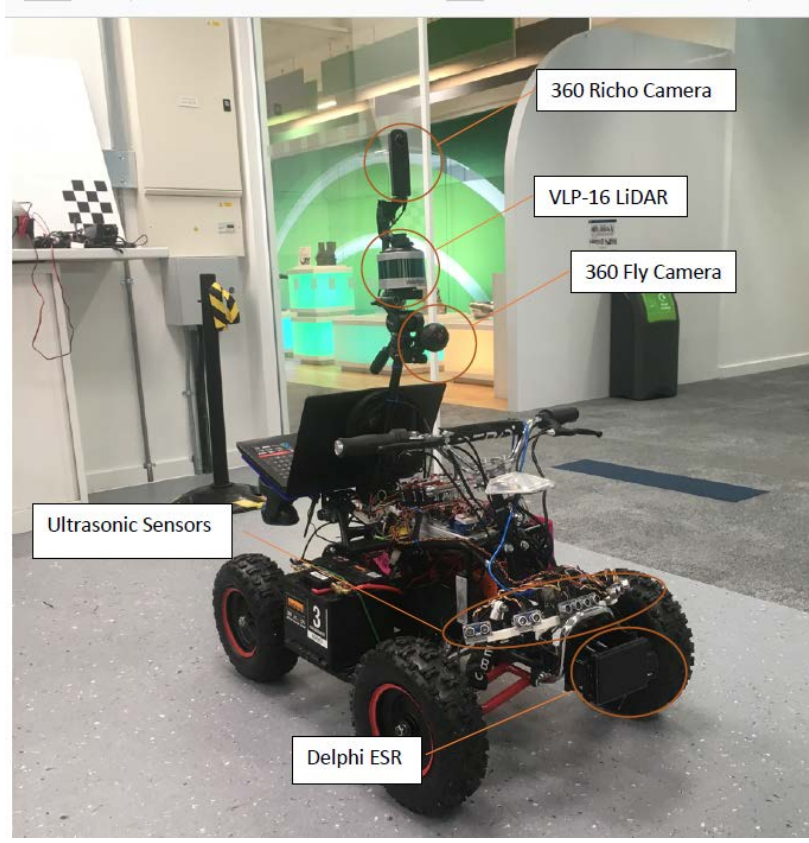

Fig. 4. The experimental Test bet for Intelligent Mobility

\section{Personal Assistive Robot System Development}

In this section we will describe how to integrate a mobile robot with the ambient intelligence architecture proposed in section III. This section sets out a futuristic vision for the intelligent control of robots with the aid of IoT.

\section{A. Robot Integration with Ambient Intelligence Framework}

A mobile robot will utilize a multitude of sensors to perceive and understand its environment. These sensors include, cameras, ultrasound sensors, LiDARs and Radars. The building blocks of a typical indoor mobile robot and its integration to the ambient intelligence framework is illustrated in Fig. 3.

To address the challenges of indoor navigation, the robot will communicate with the context gathering system through wireless communication. The location dependent context communicated by the system will provide information about location of human subjects, human activities and obstacles. The context information can then be utilized by robot navigation control system to localize itself and then to take navigation decisions such acceleration, braking and steering control.

Scalability of the context gathering framework is utilized in several ways: while the robot can get obstacle information surrounding it, depending on the steering controls high resolution information can be acquired. At the same time, the robot senses high resolution 3-dimensional environmental context through its sensors and communicates with the context gathering layer to update the centrally stored context layers.

\section{B. Description of the Test bed}

An experimental test bed was constructed to demonstrate various applications related to intelligent mobility. The overall objective was to develop an autonomous test bed that could traverse both indoors and outdoors alike. We expect to

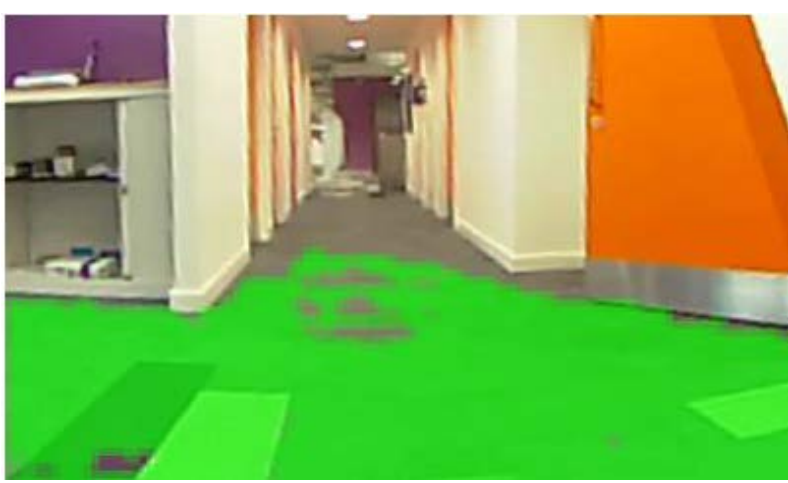

Fig. 5. Free Space Detection: Initial results: Green color indicates areas that are void of obstacles

utilize this robot to investigate research questions related to autonomous driving and assistive robots such as robust navigation and free space detection, multimodal data collection and fusion and adaptive control algorithms.

The developed test bed is illustrated in Fig. 4. The test bed is composed of a front facing wide angle camera, a rear facing camera, and a LiDAR scanner tagged to an electric quad bike. However, in this paper we are focused on fusing only the front facing wide angle camera output with the LiDAR scanner output.

The Velodyne VLP-16 LiDAR, which is used in the test bed, a compact low power light-weight optical sensor, has a maximum range of 100 metres. The sensor supports 16 channel communications taking a total of 300,000 measurements per second. Data is captured over $360^{\circ}$ on the horizontal axis and $30^{\circ}$ on the vertical, utilizing 16 laser/detector pairs.

The wide angle camera utilized in the setup is a 360Fly camera that is enclosed in a $61 \mathrm{~mm}$ diameter sphere with a single fish eye lens mounted on the top. The field of view is $360^{\circ}$ on the vertical and $240^{\circ}$ horizontal. Standard $360^{\circ}$ video that is output from this camera is a flat equi-rectangular video displayed as a sphere.

Our initial tests have shown that ...

To demonstrate the benefits of the ambient intelligence architecture proposed in this paper we will further our initial research on free space detection. Free space detection as illustrated in Fig. 5 is to detect areas that are obstacle free. We will use this platform to demonstrate robust approaches for free space detection, and localization of the robot in indoor environments.

\section{CONCLUSIONS AND FUTURE WORK}

The wide spread availability of Internet of Things has enabled to gather complex multimodal context of environments. This paper proposed a scalable multi-layered mapping architecture to represent various context gathered through advanced analysis of IoT sensor data to facilitate ambient intelligence to cater various adaptive control algorithms. This paper particularly focused on personal assistive robots and discussed how to address the challenges of robot navigation and localization in complex and dynamic indoor environments. Future work will be to investigate the .... using our in-house built fully autonomous test bed in a controlled environment with mobile obstacles. 


\section{REFERENCES}

[1] K. E. C. Booth, S. C. Mohamed, S. Rajaratnam, G. Nejat, and J. C. Beck, "Robots in Retirement Homes: Person Search and Task Planning for a Group of Residents by a Team of Assistive Robots,” IEEE Intelligent Systems, vol. 32, no. 6, pp. 14-21, Nov. 2017.

[2] M. Martinez, A. Roitberg, D. Koester, R. Stiefelhagen, and B. Schauerte, "Using Technology Developed for Autonomous Cars to Help Navigate Blind People," in 2017 IEEE International Conference on Computer Vision Workshops (ICCVW), 2017, pp. 1424-1432.

[3] S. H. Chen et al., "Assistive Control System for Upper Limb Rehabilitation Robot,” IEEE Transactions on Neural Systems and Rehabilitation Engineering, vol. 24, no. 11, pp. 11991209, Nov. 2016.

[4] J. Paulo, P. Peixoto, and U. J. Nunes, "ISR-AIWALKER: Robotic Walker for Intuitive and Safe Mobility Assistance and Gait Analysis,” IEEE Transactions on Human-Machine Systems, vol. 47, no. 6, pp. 1110-1122, Dec. 2017.

[5] A. Jevtić et al., "Personalized Robot Assistant for Support in Dressing," IEEE Transactions on Cognitive and Developmental Systems, pp. 1-1, 2018.

[6] D. Park, Y. Hoshi, and C. C. Kemp, "A Multimodal Anomaly Detector for Robot-Assisted Feeding Using an LSTM-Based Variational Autoencoder," IEEE Robotics and Automation Letters, vol. 3, no. 3, pp. 1544-1551, Jul. 2018.

[7] E. Martinez-Martin and A. P. del Pobil, "Object Detection and Recognition for Assistive Robots: Experimentation and Implementation,” IEEE Robotics Automation Magazine, vol. 24, no. 3, pp. 123-138, Sep. 2017.

[8] M. Ficocelli, J. Terao, and G. Nejat, "Promoting Interactions Between Humans and Robots Using Robotic Emotional Behavior," IEEE Transactions on Cybernetics, vol. 46, no. 12, pp. 2911-2923, Dec. 2016.

[9] P. T. Jardine and S. N. Givigi, "A Robust Model-Predictive Guidance System for Autonomous Vehicles in Cluttered Environments,” IEEE Systems Journal, pp. 1-12, 2018.

[10] M. A. V. J. Muthugala and A. G. B. P. Jayasekara, "Enhancing User Satisfaction by Adapting Robot \#x2019; Perception of Uncertain Information Based on Environment and User Feedback,” IEEE Access, vol. 5, pp. 26435-26447, 2017.

[11] O. B. Sezer, E. Dogdu, and A. M. Ozbayoglu, "ContextAware Computing, Learning, and Big Data in Internet of Things: A Survey," IEEE Internet of Things Journal, vol. 5, no. 1, pp. 1-27, Feb. 2018.
[12] P. Fonseka and K. Sandrasegaran, "Indoor localization for IoT applications using fingerprinting," in 2018 IEEE 4th World Forum on Internet of Things (WF-IoT), 2018, pp. 736-741.

[13] S. Han, Y. Li, W. Meng, C. Li, T. Liu, and Y. Zhang, "Indoor Localization With a Single Wi-Fi Access Point Based on OFDM-MIMO,” IEEE Systems Journal, pp. 1-9, 2018.

[14] T. Wenge, M. T. Chew, F. Alam, and G. S. Gupta, "Implementation of a visible light based indoor localization system," in 2018 IEEE Sensors Applications Symposium (SAS), 2018, pp. 1-6.

[15] H. Zheng, Z. Xu, C. Yu, and M. Gurusamy, "A 3-D high accuracy positioning system based on visible light communication with novel positioning algorithm," Optics Communications, vol. 396, pp. 160-168, Aug. 2017.

[16] S. Y. Jung, S. Hann, and C. S. Park, "TDOA-based optical wireless indoor localization using LED ceiling lamps," IEEE Transactions on Consumer Electronics, vol. 57, no. 4, pp. 1592-1597, Nov. 2011.

[17] R. Leonardo, M. Barandas, and H. Gamboa, “A Framework for Infrastructure-Free Indoor Localization Based on Pervasive Sound Analysis,” IEEE Sensors Journal, vol. 18, no. 10, pp. 4136-4144, May 2018.

[18] A. Popleteev, "Please stand by: TV-based indoor localization," in 2017 IEEE 28th Annual International Symposium on Personal, Indoor, and Mobile Radio Communications (PIMRC), 2017, pp. 1-6.

[19] X. Guo, N. Ansari, L. Li, and H. Li, "Indoor Localization by Fusing a Group of Fingerprints Based on Random Forests," IEEE Internet of Things Journal, pp. 1-1, 2018.

[20] Z. Hu, G. Huang, Y. Hu, and Z. Yang, "WI-VI fingerprint: WiFi and vision integrated fingerprint for smartphone-based indoor self-localization,” in 2017 IEEE International Conference on Image Processing (ICIP), 2017, pp. 44024406.

[21] Q. Pu, S. Gupta, S. Gollakota, and S. Patel, "Whole-home Gesture Recognition Using Wireless Signals," in Proceedings of the 19th Annual International Conference on Mobile Computing \& Networking, New York, NY, USA, 2013, pp. 27-38.

[22] F. Adib and D. Katabi, "See Through Walls with WiFi!," in Proceedings of the ACM SIGCOMM 2013 Conference on SIGCOMM, New York, NY, USA, 2013, pp. 75-86.

[23] Y. Gu, F. Ren, and J. Li, "PAWS: Passive Human Activity Recognition Based on WiFi Ambient Signals,” IEEE Internet of Things Journal, vol. 3, no. 5, pp. 796-805, Oct. 2016.

[24] S. Yousefi, H. Narui, S. Dayal, S. Ermon, and S. Valaee, “A Survey on Behavior Recognition Using WiFi Channel State Information,” IEEE Communications Magazine, vol. 55, no. 10, pp. 98-104, Oct. 2017. 